Research Article

\title{
Regulator-Based Risk Statistics for Portfolios
}

\author{
Xiaochuan Deng ${ }^{1}$ and Fei Sun $\mathbb{D}^{2}$ \\ ${ }^{1}$ School of Economics and Management, Wuhan University, Wuhan 430072, China \\ ${ }^{2}$ School of Mathematics and Computational Science, Wuyi University, Jiangmen 529020, China
}

Correspondence should be addressed to Fei Sun; fsun.sci@outlook.com

Received 30 May 2020; Accepted 20 June 2020; Published 9 July 2020

Academic Editor: Jia-Bao Liu

Copyright (c) 2020 Xiaochuan Deng and Fei Sun. This is an open access article distributed under the Creative Commons Attribution License, which permits unrestricted use, distribution, and reproduction in any medium, provided the original work is properly cited.

Risk statistic is a critical factor not only for risk analysis but also for financial application. However, the traditional risk statistics may fail to describe the characteristics of regulator-based risk. In this paper, we consider the regulator-based risk statistics for portfolios. By further developing the properties related to regulator-based risk statistics, we are able to derive dual representation for such risk.

\section{Introduction}

Risk measure is a popular topic in both financial application and theoretical research. The quantitative calculation of risk involves two problems: choosing an appropriate risk model and allocating risk to individual institutions. This has led to further research on risk statistics. In a seminal paper, Sun et al. and Liu et al. [1,2] first introduced the class of natural risk statistics with representation results. Furthermore, Ahmed et al. [3] derived an alternative proof for the natural risk statistics. Later, Tian and Jiang and Tian and Suo $[4,5]$ obtained the representation results for convex risk statistics and quasiconvex risk statistics, respectively.

However, traditional risk statistics may fail to describe the characteristics of regulator-based risk. Therefore, the study of regulator-based risk statistics is particularly interesting. On the other hand, in the abovementioned research on risk statistics, the set-valued risk was never studied. Jouini et al. [6] pointed out that a set-valued risk measure is more appropriate than a scalar risk measure especially in the case where several different kinds of currencies are involved when one is determining capital requirements for the portfolio. Indeed, a natural set-valued risk statistic can be considered as an empirical (or a databased) version of a set-valued risk measure. More recent studies of set-valued risk measures include those of [7-14] and the references therein.

The main focus of this paper is regulator-based risk statistics for portfolios. In this context, both empirical versions and data-based versions of regulator-based risk measures are discussed. By further developing the properties related to regulator-based risk statistics, we are able to derive their dual representations. Indeed, this class of risk statistics can be considered as an extension of those introduced in [15-17].

The remainder of this paper is organized as follows. In Section 2, we briefly introduce some preliminaries. In Section 3, we state the main results of regulator-based risk statistics, including the dual representations. Section 4 investigates the data-based versions of regulator-based risk measures. Finally, in Section 5, the main proofs in this paper are discussed.

\section{Preliminary Information}

In this section, we briefly introduce some preliminaries that are used throughout this paper. Let $d \geq 1$ be a fixed positive integer. The space $\mathbb{R}^{d \times n}$ represents the set of financial risk positions. With positive values of $X \in \mathbb{R}^{d \times n}$, we denote the gains while the negative denotes the losses. Let $n_{j}$ be the sample size of $D=\left(X_{1}, \ldots, X_{d}\right)$ in the $j^{\text {th }}$ scenario, $j=1, \ldots, l$. Let $n:=n_{1}+\cdots+n_{l}$. More precisely, suppose that the behavior of $D$ is represented by a collection of data $X=\left(X_{1}, \ldots, X_{d}\right) \in \mathbb{R}^{n} \times \cdots \times \mathbb{R}^{n}$, where $X_{i}=$ $\left(X^{i, 1}, \ldots, X^{i, l}\right) \in \mathbb{R}^{n}$ and $X^{i, j}=\left(x_{1}^{i, j}, \ldots, x_{n_{j}}^{i, j}\right) \in \mathbb{R}^{n_{j}}$ is the data subset that corresponds to the $j^{\text {th }}$ scenario with respect to $X_{i}$. For each $j=1, \ldots, l, \quad h=1, \ldots, n_{j}, \quad X_{h}^{j}:=$ 
$\left(x_{h}^{1, j}, x_{h}^{2, j}, \ldots, x_{h}^{d, j}\right)$ is the data subset that corresponds to the $h^{\text {th }}$ observation of $D$ in the $j^{\text {th }}$ scenario.

In this paper, an element $z$ of $\mathbb{R}^{d}$ is denoted by $z:=\left(z_{1}, \ldots, z_{d}\right)$. An element $X$ of $\mathbb{R}^{d \times n}$ is denoted by $X:=\left(X_{1}, \ldots, X_{d}\right):=\left(x_{1}^{1,1}, \ldots, x_{n_{1}}^{1,1}, \ldots, x_{1}^{1, l}, \ldots, x_{n_{l}}^{1, l}, \ldots\right.$, $\left.x_{1}^{d, 1}, \ldots x_{n_{1}}^{d, 1}, \ldots, x_{1}^{d, l}, \ldots, x_{n_{l}}^{d, l}\right)$. Let $K$ be a closed convex polyhedral cone of $\mathbb{R}^{d}$ where $K \supseteq \mathbb{R}_{++}^{d}:=\left\{\left(x_{1}, \ldots, x_{d}\right) \in\right.$ $\left.\mathbb{R}^{d} ; x_{i}>0,1 \leq i \leq d\right\}$ and $K \cap \mathbb{R}_{-}^{d}=\varnothing$ where $\mathbb{R}_{-}^{d}:=$ $\left\{\left(x_{1}, \ldots, x_{d}\right) \in \mathbb{R}^{d} ; x_{i} \leq 0,1 \leq i \leq d\right\}$. Let $K^{+}$be the positive dual cone of $K$, that is, $K^{+}:=\left\{u \in \mathbb{R}^{d}: u^{\operatorname{tr}} v \geq 0\right.$ for any $v \in K\}$, where $u^{\text {tr }}$ means the transpose of $u$. For any $X=\left(X_{1}, \ldots, X_{d}\right), Y=\left(Y_{1}, \ldots, Y_{d}\right) \in \mathbb{R}^{d \times n}, X+Y$ stands for $\left(X_{1}+Y_{1}, \ldots, X_{d}+Y_{d}\right)$ and $a X$ stands for $\left(a X_{1}, \ldots, a X_{d}\right)$ for $a \in \mathbb{R}$. Denote $K 1_{n}:=\left\{\left(z_{1} 1_{n}, z_{2} 1_{n}, \ldots\right.\right.$, $\left.\left.z_{d} 1_{n}\right): z \in K\right\}$ and $z 1_{n}:=\{(z, z, \ldots, z): z \in \mathbb{R}\} \in \mathbb{R}^{n}$ where $1_{n}:=(1, \ldots, 1) \in \mathbb{R}^{n}$. By $\left(K 1_{n}\right)^{+}$, and we denote the positive dual cone of $K 1_{n}$ in $\mathbb{R}^{d \times n}$, i.e., $\left(K 1_{n}\right)^{+}:=$ $\left\{w \in \mathbb{R}^{d \times n}: w z^{\text {tr }} \geq 0\right.$ for any $\left.z \in K\right\}$. The partial order with respect to $K$ is defined as $a \leq_{K} b$, which means $b-a \in K$ where $a, b \in \mathbb{R}^{d}$, and $X \leq_{K 1_{n}} Y$ means $Y-X \in K 1_{n}$ where $X, Y \in \mathbb{R}^{d \times n}$.

Let $M:=\mathbb{R}^{m} \times\{0\}^{d-m}$ be the linear subspace of $\mathbb{R}^{d}$ for $1 \leq m \leq d$. The introduction of $M$ was considered in $[6,9]$. Denote $\quad M_{+}:=M \cap \mathbb{R}_{+}^{d}$, where $\mathbb{R}_{+}^{d}:=\left\{\left(x_{1}, \ldots, x_{d}\right) \in\right.$ $\left.\mathbb{R}^{d} ; x^{i} \geq 0,1 \leq i \leq d\right\}$ and $M^{\perp}:=\{0\}^{m} \times \mathbb{R}^{d-m}$. Therefore, a regulator can only accept security deposits in the first $m$ reference instruments. Denoting $K_{M}:=K \cap M$ by the closed convex polyhedral cone in $M, K_{M}^{+}:=$ $\left\{u \in M: u^{\operatorname{tr}} z \geq 0\right.$ for any $\left.z \in K_{M}\right\}$ the positive dual cone of $K_{M}$ in $M$ and int $K_{M}$ is the interior of $K_{M}$ in $M$. We denote $Q_{M}^{t}:=\left\{A \subset M: A=\operatorname{clco}\left(A+K_{M}\right)\right\}$ and $Q_{M^{+}}^{t}:=\left\{A \subset K_{M}\right.$ $\left.: A=\operatorname{clco}\left(A+K_{M}\right)\right\}$, where the $\operatorname{clco}(A)$ represents the closed convex hull of $A$.

By [18], a set-valued risk statistic is any map $\rho$,

$$
\rho: \mathbb{R}^{d \times n} \longrightarrow 2^{M}
$$

which can be considered as an empirical (or a data-based) version of a set-valued risk measure. The axioms related to this set-valued risk statistic are organized as follows:

[A0] Normalization: $K_{M} \subseteq \rho(0)$ and $\rho(0) \cap-\operatorname{int} K_{M}=\phi$

[A1] Monotonicity: for any $X, Y \in \mathbb{R}^{d \times n}, X-Y \in K 1_{n}$ implies that $\rho(X) \supseteq \rho(Y)$

[A2] M-translative invariance: for any $X \in \mathbb{R}^{d \times n}$ and $z \in \mathbb{R}^{d}, \rho\left(X-z 1_{n}\right)=\rho(X)+z$

[A3] Convexity: for any $X, Y \in \mathbb{R}^{d \times n}$ and $\lambda \in[0,1]$, $\rho(\lambda(X)+(1-\lambda) Y) \supseteq \lambda \rho(X)+(1-\lambda) \rho(Y)$

[A4] Positive homogeneity: $\rho(\lambda X)=\lambda \rho(X)$ for any $X \in \mathbb{R}^{d \times n}$ and $\lambda>0$

[A5] Subadditivity: $\rho(X+Y) \supseteq \rho(X)+\rho(Y)$ for any $X, Y \in \mathbb{R}^{d \times n}$

We end this section with more notations. A function $\rho: \mathbb{R}^{d \times n} \longrightarrow 2^{M}$ is said to be proper if $\operatorname{dom} \rho:=\left\{X \in \mathbb{R}^{d \times n}: \rho(X) \neq \varnothing\right\} \neq \varnothing$ and $\rho(X) \neq M$ for all $X \in \operatorname{dom} \rho . \rho$ is said to be closed if graph $\rho$ is a closed set. For the properties of the graphs, see [19-21].

\section{Empirical Versions of Regulator-Based Risk Measures}

In this section, we state the dual representations of regulatorbased risk statistics, which are the empirical versions of regulator-based risk measures. Firstly, for any $X \in \mathbb{R}^{d \times n}$, $X \wedge_{K 1_{n}} 0$ is defined as follows:

$$
X \wedge_{K 1_{n}} 0:= \begin{cases}X, & X \notin K 1_{n}, \\ 0, & X \in K 1_{n} .\end{cases}
$$

Therefore, the position that belongs to $K$ regarded is as 0 position. Next, we derive the properties related to regulatorbased risk statistics.

Definition 1. A regulator-based risk statistic is a function $\varrho: \mathbb{R}^{d \times n} \longrightarrow Q_{M^{+}}^{t}$ that satisfies the following properties:

[P0] Normalization: $K_{M} \subseteq \varrho(0)$ and $\varrho(0) \cap-\operatorname{int} K_{M}=\phi$

[P1] Cash cover: for any $z \in K_{M}, z \in \varrho\left(-z 1_{n}\right)$

[P2] Monotonicity: for any $X, Y \in \mathbb{R}^{d \times n}$, $X-Y \in \mathbb{R}^{d \times n} \cap K 1_{n}$ implies that $\varrho(X) \supseteq \varrho(Y)$

[P3] Regulator-dependence: for any $X \in \mathbb{R}^{d \times n}$, $\varrho(X)=\varrho\left(X \wedge_{K 1_{n}} 0\right)$

[P4] Convexity: for any $X, Y \in \mathbb{R}^{d \times n}$ and $\lambda \in[0,1]$, $\varrho(\lambda(X)+(1-\lambda) Y) \supseteq \lambda \varrho(X)+(1-\lambda) \varrho(Y)$

Remark 1 . The property of $[P 1]$ means any fixed negative risk position $-z$ can be canceled by its positive quality $z$; [P2] says that if $X_{1}$ is bigger than $X_{2}$ for the partial order in $K$, then the $X_{1}$ need less capital requirement than $X_{2}$, so $\varrho\left(X_{1}\right)$ contains $\varrho\left(X_{2}\right)$; $[P 3]$ means the regulator-based risk statistics start only from the viewpoint of regulators which only care about the positions that need to pay capital requirements, while the positions that belong to $K$ regarded as 0 position.

We now construct an example for regulator-based risk statistics.

Example 1. The coherent risk measure AV@R was studied by Föllmer and Schied [22] in detail. They have given several representations and many properties such as law invariance and the Fatou property [12]. First, they introduced setvalued $A V @ R$, where the representation result is derived. Moveover, they also proved that it is a set-valued coherent risk measure. We now define the regulator-based average value at risk. For any $X \in \mathbb{R}^{d \times n}$ and $0<\alpha<1$, we define $\varrho(X)$ as

$$
\begin{aligned}
\varrho(X) & :=A V @ R_{\alpha}^{\operatorname{loss}}(X), \\
& :=\inf _{z \in \mathbb{R}^{d}}\left\{\frac{1}{\alpha}\left(-\left.\left(X \wedge_{K 1_{n}} 0\right)\right|_{M}+z\right)^{+}-z\right\}+\mathbb{R}_{+}^{m},
\end{aligned}
$$

It is clear that $\varrho$ satisfies the cash cover, monotonicity, regulator dependence properties, and convexity, so $\varrho$ is a regulator-based risk statistic. 
Definition 2. Let $Y \in \mathbb{R}^{d \times n}, u \in M$. Define a function $S_{(Y, u)}(X): \mathbb{R}^{d \times n} \longrightarrow 2^{M}$ as

$$
S_{(Y, u)}(X):=\left\{z \in M: X^{\operatorname{tr}} Y \leq u^{\operatorname{tr}} z\right\} .
$$

In fact, the $S_{(Y, u)}(X)$ is the support function of $X$. Before we derive the dual representations of regulator-based risk statistics, the Legendre-Fenchel conjugate theory ([9]) should be recalled.

Lemma 1 (see [9], Theorem 2). Let $R: \mathbb{R}^{d \times n} \longrightarrow Q_{M}^{t}$ be a set-valued closed convex function. Then, the Legendre-Fenchel conjugate and the biconjugate of $R$ can be defined, respectively, as

$$
\begin{gathered}
-R^{*}(Y, u):=c l \bigcup_{X \in \mathbb{R}^{d \times n}}\left(R(X)+S_{(Y, u)}(-X)\right), \\
Y \in \mathbb{R}^{d \times n}, u \in \mathbb{R}^{d}, \\
R(X)=R^{* *}(X):={\underset{(Y, u) \in \mathbb{R}^{d \times n} \times K_{M}^{+} \backslash\{0\}}{\bigcap}}\left[-R^{*}(Y, u)+S_{(Y, u)}(X)\right], \quad X \in \mathbb{R}^{d \times n} .
\end{gathered}
$$

Definition 3 (indicator function). For any $Z \subseteq \mathbb{R}^{d \times n}$, the $Q_{M}^{t}$-valued indicator function $I_{Z}: \mathbb{R}^{d \times n} \longrightarrow Q_{M}^{t}$ is defined as

$$
I_{Z}(X):= \begin{cases}c l K_{M}, & X \in Z, \\ \phi, & X \notin Z .\end{cases}
$$

Remark 2. The conjugate of $Q_{M}^{t}$-valued indicator function $I_{Z}$ is

$$
-\left(I_{Z}\right)^{*}(Y, u):=c l \bigcup_{X \in Z} S_{(Y, u)}(-X) .
$$

Remark 3. It is easy to see that the regulator-based risk statistic $\varrho$ does not have cash additivity, see [9]. However, $\varrho$ has cash subadditivity introduced in [23, 24]. Indeed, from
Theorem 2 of [10], $\varrho$ satisfies the Fatou property. Then, considering any $X \in \mathbb{R}^{d \times n}$ and $z \in K_{M}$, for any $\varepsilon \in(0,1)$, we have

$$
\begin{aligned}
\varrho\left((1-\varepsilon) X-z 1_{n}\right) & =\varrho\left((1-\varepsilon) X+\varepsilon\left(-\frac{z}{\varepsilon}\right) 1_{n}\right), \\
& \supseteq(1-\varepsilon) \varrho(X)+\varepsilon \varrho\left(-\frac{z}{\varepsilon} 1_{n}\right), \\
& \supseteq(1-\varepsilon) \varrho(X)+z,
\end{aligned}
$$

where the last inclusion is due to the property $[P 1]$. Using the arbitrariness of $\varepsilon$, we have the following lemma.

Lemma 2. Assume that $\mathrm{Q}$ is a regulator-based risk statistic. For any $z \in \mathbb{R}_{+}^{d}, X \in \mathbb{R}^{d \times n}$,

$$
\varrho\left(X-z 1_{n}\right) \supseteq \varrho(X)+z,
$$

which also implies

$$
\varrho\left(X+z 1_{n}\right) \subseteq \varrho(X)-z .
$$

Proposition 1. Let $\varrho: \mathbb{R}^{d \times n} \longrightarrow Q_{M^{+}}^{t}$ be a proper closed convex regulator-based risk statistic with $u \in\left\{\left(-\sum_{j=1}^{l} \sum_{h=1}^{n_{j}} Y_{h}^{1, j}, \ldots,-\sum_{j=1}^{l} \sum_{h=1}^{n_{j}} Y_{h}^{d, j}\right)+M^{\perp}\right\} \cap K_{M}^{+} \backslash$ $\{0\}$. Then,

$-\varrho^{*}(Y, u)= \begin{cases}c l \bigcup_{X \in \mathbb{R}^{d \times n}} S_{(Y, u)}(-X), & \mathrm{Y} \in-\mathbb{R}_{+}^{d \times n} \cap\left(K^{+} 1_{n}\right), \\ M, & \text { elsewhere. }\end{cases}$

Now, we state the main result of this paper, the dual representations of regulator-based risk statistics.

Theorem 1. If $\varrho: \mathbb{R}^{d \times n} \longrightarrow Q_{M^{+}}^{t}$ is a proper closed convex regulator-based risk statistic, then there is a $-\alpha:\left(-\mathbb{R}_{+}^{d \times n} \cap K^{+} 1_{n}\right) \times K_{M}^{+}\{0\} \longrightarrow Q_{M^{+}}^{t}$, which is not identically $M$ of the set

$$
\mathrm{W}=\left\{(Y, u) \in\left(-\mathbb{R}_{+}^{d \times n} \cap K^{+} 1_{n}\right) \times K_{M}^{+} \backslash\{0\} u \in\left(-\sum_{j=1}^{l} \sum_{h=1}^{n_{j}} Y_{h}^{1, j}, \ldots,-\sum_{j=1}^{l} \sum_{h=1}^{n_{j}} Y_{h}^{d, j}\right)+M^{\perp}\right\},
$$

such that for any $X \in \mathbb{R}^{d \times n}$,

$$
\varrho(X)=\underset{(Y, u) \in \mathrm{W}}{\cap}\left\{-\alpha(Y, u)+S_{(Y, u)}\left(X \wedge_{K 1_{n}} 0\right)\right\} .
$$

\section{Alternative Data-Based Versions of Regulator-Based Risk Measures}

In this section, we develop another framework, the data-based versions of regulator-based risk measures. This framework is a little different from the previous one. However, almost all the arguments are the same as those in the previous section.
Therefore, we only state the corresponding notations and results and omit all the proofs and relevant explanations.

We replace $M$ by $\widetilde{M} \in \mathbb{R}^{d \times n}$ that is a linear subspace of $\mathbb{R}^{d \times n}$. We also replace $K$ by $\widetilde{K} \in \mathbb{R}^{d \times n}$ that is a is a closed convex polyhedral cone where $\widetilde{K} \supseteq \mathbb{R}_{++}^{d \times n}$. The partial order with respect to $\widetilde{K}$ is defined as $X \leq \widetilde{K} Y$, which means $Y-X \in \widetilde{K}$. Let $\widetilde{M}_{+}:=\widetilde{M} \cap \mathbb{R}_{+}^{d \times n}$. Denoting $\widetilde{K}_{\widetilde{M}}:=\widetilde{K} \cap \widetilde{M}$ by the closed convex polyhedral cone in $\tilde{M}$, $\widetilde{K}_{\widetilde{M}}^{+}:=\left\{\widetilde{u} \in M: \widetilde{u}^{\operatorname{tr}} \widetilde{z} \geq 0\right.$ for any $\left.\widetilde{z} \in \widetilde{K}_{\widetilde{M}}\right\}$ is the positive dual cone of $\widetilde{K}_{\widetilde{M}}$ in $\widetilde{M}$ and int $\widetilde{K}_{\widetilde{M}}$ is the interior of $\widetilde{K}_{\widetilde{M}}$ in $\widetilde{M}$. We denote $\quad Q_{\widetilde{M}}^{t}:=\left\{\widetilde{A} \subset \widetilde{M}: \widetilde{A}=\operatorname{clco}\left(\widetilde{A}+\widetilde{K}_{\widetilde{M}}\right)\right\} \quad$ and 
$Q_{\widetilde{M}^{+}}^{t}:=\left\{\widetilde{A} \subset \widetilde{K}_{\widetilde{M}}: \widetilde{A}=\operatorname{clco}\left(\widetilde{A}+\widetilde{K}_{\widetilde{M}}\right)\right\}$. We still start from the viewpoint of regulators which only care about the positions that need to pay capital requirements. Therefore, for any $X \in \mathbb{R}^{d \times n}$, we define $X \wedge \widetilde{K}^{0} 0$ as

$$
X \wedge \widetilde{K}^{0}:= \begin{cases}X, & X \notin \widetilde{K}, \\ 0, & X \in \widetilde{K},\end{cases}
$$

Then, we state the axioms related to regulator-based risk statistics.

Definition 4. A regulator-based risk statistic is a function $\widetilde{\varrho}: \mathbb{R}^{d \times n} \longrightarrow Q_{\widetilde{M}^{+}}^{t}$ that satisfies the following properties:

[Q0] Normalization: $\widetilde{K}_{\widetilde{M}} \subseteq \widetilde{\varrho}(0)$ and $\widetilde{\varrho}(0) \cap-\operatorname{int} \widetilde{K}_{\widetilde{M}}=\phi$

[Q1] Cash cover: for any $\widetilde{z} \in \widetilde{K}_{\widetilde{M}}, \widetilde{z} \in \widetilde{\varrho}(-\widetilde{z})$

[Q2] Monotonicity: for any $X_{1}, X_{2} \in \mathbb{R}^{d \times n}$, $X_{1}-X_{2} \in \mathbb{R}^{d \times n} \cap \widetilde{K}$ implies that $\widetilde{\varrho}\left(X_{1}\right) \supseteq \widetilde{\varrho}\left(X_{2}\right)$

[Q3] Regulator-dependence: for any $X \in \mathbb{R}^{d \times n}$, $\widetilde{\varrho}(X)=\widetilde{\varrho}\left(X \wedge \widetilde{K}^{0}\right)$

[Q4] Convexity: for any $X, Y \in \mathbb{R}^{d \times n}, \quad \lambda \in[0,1]$, $\widetilde{\varrho}(\lambda(X)+(1-\lambda) Y) \supseteq \lambda \widetilde{\varrho}(X)+(1-\lambda) \widetilde{\varrho}(Y)$

We need more notations. Let $Y \in \mathbb{R}^{d \times n}, \tilde{u} \in \tilde{M}$. Define a function $S_{(Y, \widetilde{u})}(X): \mathbb{R}^{d \times n} \longrightarrow 2^{M}$ as

$$
S_{(Y, \widetilde{u})}(X):=\left\{\tilde{z} \in \tilde{M}: X^{\operatorname{tr}} Y \leq \widetilde{u}^{\operatorname{tr}} \widetilde{z}\right\},
$$

let $\widetilde{R}: \mathbb{R}^{d \times n} \longrightarrow Q_{\widetilde{M}}^{t}$ be a set-valued closed convex function. Then, the Legendre-Fenchel conjugate and the biconjugate of $\widetilde{R}$ can be defined, respectively, as

$$
\begin{gathered}
-\widetilde{R}^{*}(Y, u):=c l \bigcup_{X \in \mathbb{R}^{d \times n}}\left(\widetilde{R}(X)+S_{(Y, \widetilde{u})}(-X)\right), \\
Y \in \mathbb{R}^{d \times n}, \widetilde{u} \in \mathbb{R}^{d \times n}, \\
\widetilde{R}(X)=\widetilde{R}^{* *}(X):=\bigcap_{(Y, \widetilde{u}) \in \mathbb{R}^{d \times n} \times \widetilde{K}_{M}^{ \pm} \backslash\{0\}} \widetilde{R}_{\left(-\widetilde{R}^{*}(Y, \widetilde{u})+S_{(Y, \widetilde{u})}(X)\right], \quad X \in \mathbb{R}^{d \times n} .}
\end{gathered}
$$

For any $\widetilde{Z} \subseteq \mathbb{R}^{d \times n}$, the $Q_{\widetilde{M}}^{t}$-valued indicator function $I_{\widetilde{Z}}: \mathbb{R}^{d \times n} \longrightarrow Q_{\widetilde{M}}^{t}$ is defined as

$$
I_{\widetilde{Z}}(X):= \begin{cases}c l \widetilde{K}_{\tilde{M}}, & X \in \widetilde{Z}, \\ \phi, & X \notin \widetilde{Z} .\end{cases}
$$

The conjugate of $Q_{\widetilde{M}}^{t}$-valued indicator function $I_{\widetilde{Z}}$ is

$$
-\left(I_{\tilde{Z}}\right)^{*}(Y, \widetilde{u}):=c l \bigcup_{X \in \widetilde{Z}} S_{(Y, \widetilde{u})}(-X) .
$$

Assume that $\widetilde{\varrho}$ is a regulator-based risk statistic. For any $\tilde{z} \in \mathbb{R}_{+}^{d \times n}, X \in \mathbb{R}^{d \times n}$,

$$
\widetilde{\varrho}(X-\widetilde{z}) \supseteq \widetilde{\varrho}(X)+\widetilde{z},
$$

which also implies

$$
\widetilde{\varrho}(X+\widetilde{z}) \subseteq \widetilde{\varrho}(X)-\widetilde{z} .
$$

Next, we state the dual representations of regulatorbased risk statistics.

Proposition 2. Let $\widetilde{\varrho}: \mathbb{R}^{d \times n} \longrightarrow Q_{\widetilde{M}^{+}}^{t}$ be a proper closed convex regulator-based risk statistic ${ }^{M}$ with $\widetilde{u} \in\left\{\left(-\sum_{j=1}^{l} \sum_{h=1}^{n_{j}}\right.\right.$ $\left.\left.Y_{h}^{1, j}, \ldots,-\sum_{j=1}^{l} \sum_{h=1}^{n_{j}} Y_{h}^{d, j}\right)+\widetilde{M}^{\perp}\right\} \cap \widetilde{K}_{\widetilde{M}}^{+} \backslash\{0\}$. Then,

$$
-\widetilde{\varrho}^{*}(Y, \widetilde{u})= \begin{cases}c l \bigcup_{X \in \mathbb{R}^{d \times n}} S_{(Y, \widetilde{u})}(-X), & Y \in-\mathbb{R}_{+}^{d \times n} \cap\left(\widetilde{K}^{+}\right), \\ \tilde{M}, & \text { elsewhere. }\end{cases}
$$

Theorem 2. If $\widetilde{\varrho}: \mathbb{R}^{d \times n} \longrightarrow Q_{\widetilde{u}^{+}}^{t}$ is a proper closed convex regulator-based risk statistic, then there is a $-\alpha$ : $\left(-\mathbb{R}_{+}^{d \times n} \cap \widetilde{K}^{+}\right) \times \widetilde{K}_{\widetilde{M}}^{+} \backslash\{0\} \longrightarrow Q_{\tilde{M}^{+}}^{+}$, that is not identically $\widetilde{M}$ of
the set

$$
\widetilde{\mathrm{W}}=\left\{(Y, \widetilde{u}) \in\left(-\mathbb{R}_{+}^{d \times n} \cap \widetilde{K}^{+}\right) \times \widetilde{K}_{\widetilde{M}}^{+} \backslash\{0\}: \widetilde{u} \in\left(-\sum_{j=1}^{l} \sum_{h=1}^{n_{j}} Y_{h}^{1, j}, \ldots,-\sum_{j=1}^{l} \sum_{h=1}^{n_{j}} Y_{h}^{d, j}\right)+\widetilde{M}^{\perp}\right\}
$$

such that for any $X \in \mathbb{R}^{d \times n}$,

$$
\widetilde{\varrho}(X)=\bigcap_{(Y, \widetilde{u}) \in \mathrm{W}}\left\{-\alpha(Y, \widetilde{u})+S_{(Y, \widetilde{u})}\left(X \wedge \wedge_{\tilde{K}} 0\right)\right\} .
$$

\section{Proofs of Main Results}

Proof of Lemma 2. the proof of Lemma 2 is straightforward from Remark 3.

Proof of Proposition 1. if $Y \notin-\mathbb{R}_{+}^{d \times n} \cap\left(K^{+} 1_{n}\right)$, there exits an $\bar{X} \in \mathbb{R}^{d \times n} \cap\left(K 1_{n}\right)$ such that $\bar{X}^{\mathrm{tr}} Y>0$. Using the definition of
$S_{(Y, u)}$, we have $S_{(Y, u)}(-t \bar{X})=\left\{z \in M:-t \bar{X}^{\operatorname{tr}} Y \leq u^{\text {tr }} z\right\}$ for $t>0$. Therefore,

$$
\mathrm{cl} \underset{X \in \mathbb{R}^{d \times n}}{\cup} S_{(Y, u)}(-X) \supseteq \underset{t>0}{\cup} S_{(Y, u)}(-t \bar{X})=M .
$$

The last equality is due to $-t \bar{X}^{\mathrm{tr}} Y \longrightarrow-\infty$ when $t \longrightarrow+\infty$. Using the definition of $S_{(Y, u)}$, we conclude that $\mathrm{cl} \cup_{X \in \mathbb{R}^{d \times n} S_{(Y, u)}}(-X) \subseteq M$. Hence,

$\mathrm{cl} \underset{X \in \mathbb{R}^{d \times n}}{\cup} S_{(Y, u)}(-X)=M, \quad Y \notin-\mathbb{R}^{d \times n} \cap\left(K^{+} 1_{n}\right)$. 
It is easy to check that for any $X \in \mathbb{R}^{d \times n}$ and $v \in M$,

$$
\begin{aligned}
S_{(Y, u)}\left(-X-v 1_{n}\right) & =\left\{z \in M:-X^{\operatorname{tr}} Y \leq u^{\operatorname{tr}} z+Y^{\operatorname{tr}}\left(v 1_{n}\right)\right\} \\
& =\left\{z-v \in M:-X^{\operatorname{tr}} Y \leq u^{\operatorname{tr}}(z-v)+\left(Y+u 1_{n}\right)^{\operatorname{tr}}\left(v 1_{n}\right)\right\}+v, \\
& =\left\{z \in M:-X^{\operatorname{tr}} Y \leq u^{\operatorname{tr}} z+\left(Y+u 1_{n}\right)^{\operatorname{tr}}\left(v 1_{n}\right)\right\}+v,
\end{aligned}
$$

when $\quad\left(-\sum_{j=1}^{l} \sum_{h=1}^{n_{j}} Y_{h}^{1, j}, \ldots,-\sum_{j=1}^{l} \sum_{h=1}^{n_{j}} Y_{h}^{d, j}\right)+u \in M^{\perp}$, and we have $S_{(Y, u)}\left(-X-v 1_{n}\right)=S_{(Y, u)}(-X)+v$. However, $u \notin\left(\left(-\sum_{j=1}^{l} \sum_{h=1}^{n_{j}} Y_{h}^{1, j}, \ldots,-\sum_{j=1}^{l} \sum_{h=1}^{n_{j}} Y_{h}^{d, j}\right)+M^{\perp}\right)$. Therefore, $\left(-\sum_{j=1}^{l} \sum_{h=1}^{n_{j}} Y_{h}^{1, j}, \ldots,-\sum_{j=1}^{l} \sum_{h=1}^{n_{j}} Y_{h}^{d, j}\right)+u \notin M^{\perp}$, and we can find $v \in M$ such that for any $z \in M$,

$$
-X^{\operatorname{tr}} Y \leq u^{\operatorname{tr}} z+\left(Y+u 1_{n}\right)^{\operatorname{tr}}\left(v 1_{n}\right) .
$$

Therefore, we have

$$
z+v \in S_{(Y, u)}\left(-X-v 1_{n}\right)
$$

Therefore,

$$
\bigcup_{z, v \in M}(z+v) \subset \bigcup_{v \in M} S_{(Y, u)}\left(-X-v 1_{n}\right) .
$$

Therefore,

$$
M \subset \underset{v \in M}{\cup} S_{(Y, u)}\left(-X-v 1_{n}\right) .
$$

From the definition of $S_{(Y, u)}$, the inverse inclusion is always true. So, we conclude that

$$
M=\bigcup_{v \in M} S_{(Y, u)}\left(-X-v 1_{n}\right) .
$$

It is also easy to check that

$$
\begin{aligned}
-\varrho^{*}(Y, u) & =\mathrm{cl} \bigcup_{X \in \mathbb{R}^{d \times n}, v \in M}\left(\varrho\left(X+v 1_{n}\right)+S_{(Y, u)}\left(-X-v 1_{n}\right)\right), \\
& =\mathrm{cl} \bigcup_{X \in \mathbb{R}^{d \times n}, v \in M}\left(\varrho\left(X+v 1_{n}\right)+M\right), \\
& =M,
\end{aligned}
$$

where the last equality comes from the fact that $M$ is a linear space and $\varrho(X) \subseteq M$. We now derive that $-\varrho^{*}(Y, u)=c l \cup_{X \in \mathbb{R}^{d \times n}} S_{(Y, u)}(-X)$. In this context, from $-\varrho^{*}(Y, u)=c l \cup_{X \in \mathbb{R}^{d \times n}}\left(\varrho(X)+S_{(Y, u)}(-X)\right)$, we derive it in two cases.

Case 1. When $X \wedge_{K 1_{n}} 0=0$, using the definition, we have $\varrho(X)=\varrho(0) \ni 0$. Hence,

$$
c l \bigcup_{X \in \mathbb{R}^{d \times n}}\left(\varrho(X)+S_{(Y, u)}(-X)\right)^{c l} \underset{X \in \mathbb{R}^{d \times n}}{\cup} S_{(Y, u)}(-X) .
$$

Case 2. When $X \wedge_{K 1} 0=X$, we can always find an $\alpha \in K_{M}$ such that $\alpha \in \varrho(X)$. Then,

$$
\varrho(X)+S_{(Y, u)}(-X) \supseteq \alpha+S_{(Y, u)}(-X)=S_{(Y, u)}\left(-X-\alpha 1_{n}\right)=S_{(Y, u)}(-\beta),
$$

where $\beta=X+\alpha 1_{n}$. It is relatively simple to check that $\beta \in \mathbb{R}^{d \times n}$. Therefore,

$$
c l \bigcup_{X \in \mathbb{R}^{d \times n}}\left(\varrho(X)+S_{(Y, u)}(-X)\right) \supseteq c l \bigcup_{z \in \mathbb{R}^{d \times n}} S_{(Y, u)}(-z),
$$

that is,

$$
-\varrho^{*}(Y, u) \supseteq c l \bigcup_{X \in \mathbb{R}^{d \times n}} S_{(Y, u)}(-X) .
$$

Consequently, we have

$$
-\varrho^{*}(Y, u) \supseteq c l \bigcup_{X \in \mathbb{R}^{d \times n}} S_{(Y, u)}(-X) .
$$

We now need only to derive that $-\varrho^{*}(Y, u) \subseteq c l \cup_{X \in \mathbb{R}^{d \times n}} S_{(Y, u)}(-X)$. In fact, for any $z \in \varrho(X)$ and $X \in \mathbb{R}^{d \times n}, X+z 1_{n} \in \mathbb{R}^{d \times n}$. Therefore,

$$
\begin{aligned}
& c l \underset{X \in \mathbb{R}^{d \times n}}{\cup} S_{(Y, u)}(-X)=c l \underset{X \in \mathbb{R}^{d \times n}}{\bigcup} S_{(Y, u)}(-X) \supseteq S_{(Y, u)}\left(-X-z 1_{n}\right) \\
& =S_{(Y, u)}(-X)+z \text {. }
\end{aligned}
$$

Using the arbitrariness of $z$, we have

$$
\varrho(X)+S_{(Y, u)}(-X) \subseteq c l \bigcup_{X \in \mathbb{R}^{d \times n}} S_{(Y, u)}(-X) .
$$

Therefore,

$$
-\varrho^{*}(Y, u) \subseteq c l \underset{X \in \mathbb{R}^{d \times n}}{\bigcup} S_{(Y, u)}(-X) .
$$

Proof of Theorem 1. the proof is straightforward from Lemma 1 and Proposition 1.

\section{Data Availability}

No data and code were generated or used during the study.

\section{Disclosure}

This manuscript has been released as a preprint at arXiv: $1904.08829 v 4$.

\section{Conflicts of Interest}

The authors declare no conflicts of interest.

\section{Acknowledgments}

This work was supported by funds from Education Department of Guangdong (2019KQNCX156). 


\section{References}

[1] C. C. Heyde, S. G. Kou, and X. H. Peng, What is a Good External Risk Measure: Bridging the Gaps between Robustness, Subadditivity, and Insurance Risk Measures, Columbia University, New York City, NY, USA, 2007.

[2] S. Kou, X. Peng, and C. C. Heyde, "External risk measures and basel accords," Mathematics of Operations Research, vol. 38, no. 3, pp. 393-417, 2013.

[3] S. Ahmed, D. Filipović, and G. Svindland, "A note on natural risk statistics," Operations Research Letters, vol. 36, no. 6, pp. 662-664, 2008.

[4] D. Tian and L. Jiang, "Quasiconvex risk statistics with scenario analysis," Mathematics and Financial Economics, vol. 9, no. 2, pp. 111-121, 2015.

[5] D. Tian and X. Suo, "A note on convex risk statistic," Operations Research Letters, vol. 40, no. 6, pp. 551-553, 2012.

[6] E. Jouini, M. Meddeb, and N. Touzi, "Vector-valued coherent risk measures," Finance Stoch, vol. 8, no. 4, pp. 531-552, 2004.

[7] C. Ararat, A. H. Hamel, and B. Rudloff, "Set-valued shortfall and divergence risk measures," 2017, https://arxiv.org/abs/ 1405.4905

[8] W. Farkas, P. Koch-Medina, and C. Munari, "Measuring risk with multiple eligible assets," Mathematics and Financial Economics, vol. 9, no. 1, pp. 3-27, 2015.

[9] A. H. Hamel, "A duality theory for set-valued functions i: fenchel conjugation theory," Set-Valued and Variational Analysis, vol. 17, no. 2, pp. 153-182, 2009.

[10] A. H. Hamel and F. Heyde, "Duality for set-valued measures of risk," SIAM Journal on Financial Mathematics, vol. 1, no. 1, pp. 66-95, 2010.

[11] A. H. Hamel, F. Heyde, and B. Rudloff, "Set-valued risk measures for conical market models," Mathematics and Financial Economics, vol. 5, no. 1, pp. 1-28, 2011.

[12] A. H. Hamel, B. Rudloff, and M. Yankova, "Set-valued average value at risk and its computation," Mathematics and Financial Economics, vol. 7, no. 2, pp. 229-246, 2013.

[13] C. C. A. Labuschagne and T. M. Offwood-Le Roux, "Representations of set-valued risk measures defined on the $\$ \$ 1 \$ 1$ -tensor product of banach lattices," Positivity, vol. 18, no. 3, pp. 619-639, 2014.

[14] I. Molchanov and I. Cascos, "Multivariate risk measures: a constructive approach based on selections," Mathematical Finance, vol. 26, no. 4, pp. 867-900, 2016.

[15] Y. Chen, F. Sun, and Y. Hu, "Coherent and convex loss-based risk measures for portfolio vectors," Positivity, vol. 22, no. 1, pp. 399-414, 2018.

[16] R. Cont, R. Deguest, and X. D. He, "Loss-based risk measures," Statistics \& Risk Modeling, vol. 30, no. 2, pp. 133-167, 2013.

[17] F. Sun, Y. Chen, and Y. Hu, "Set-valued loss-based risk measures," Positivity, vol. 22, no. 3, pp. 859-871, 2018.

[18] Y. Chen and Y. Hu, "Set-valued risk statistics with scenario analysis," Statistics \& Probability Letters, vol. 131, pp. 25-37, 2017.

[19] J.-B. Liu, C. Wang, S. Wang, and B. Wei, "Zagreb indices and multiplicative zagreb indices of eulerian graphs," Bulletin of the Malaysian Mathematical Sciences Society, vol. 42, no. 1, pp. 67-78, 2019.

[20] J. Liu, J. Zhao, J. Min, and J. Cao, "On the hosoya index of graphs formed by a fractal graph," Fractals-Complex Geometry Patterns and Scaling in Nature and Society, vol. 27, no. 8, pp. 19-35, 2019.
[21] J.-B. Liu, J. Zhao, H. He, and Z. Shao, "Valency-based topological descriptors and structural property of the generalized sierpiński networks," Journal of Statistical Physics, vol. 177, no. 6, pp. 1131-1147, 2019.

[22] H. Föllmer and A. Schied, Stochastic Finance: An Introduction in Discrete Time, Walter de Gruyter Berlin, Berlin, Germany, Third edition, 2011.

[23] N. El Karoui and C. Ravanelli, "Cash subadditive risk measures and interest rate ambiguity," Mathematical Finance, vol. 19, no. 4, pp. 561-590, 2009.

[24] F. Sun and Y. Hu, "Set-valued cash sub-additive risk measures," Probability in the Engineering and Informational Sciences, vol. 33, no. 2, pp. 241-257, 2019. 